\title{
Information System Engineering Supporting Observation, Orientation, Decision, and Compliant Action
}

\author{
Dimitrios Georgakopoulos
}

Telcordia, USA

\begin{abstract}
The majority of today's software systems and organizational/business structures have been built on the foundation of solving problems via long-term data collection, analysis, and solution design. This traditional approach of solving problems and building corresponding software systems and business processes, falls short in providing the necessary solutions needed to deal with many problems that require agility as the main ingredient of their solution. For example, such agility is needed in responding to an emergency, in military command control, physical security, price-based competition in business, investing in the stock market, video gaming, network monitoring and self-healing, diagnosis in emergency health care, and many other areas that are too numerous to list here. The concept of Observe, Orient, Decide, and Act (OODA) loops is a guiding principal that captures the fundamental issues and approach for engineering information systems that deal with many of these problem areas. However, there are currently few software systems that are capable of supporting OODA. In this talk, we provide a tour of the research issues and state of the art solutions for supporting OODA. In addition, we provide specific examples of OODA solutions we have developed for the video surveillance and emergency response domains.
\end{abstract}

